\title{
Optimization on the Law of Variable-Pitch Vertical-Axis Tidal Current Turbine
}

\author{
Kong Fankai, Wang Liying, Zhang Di \\ Mechanical Engineering, Harbin Engineering University, Harbin 150001, China
}

\begin{abstract}
In order to improve the hydrodynamic performance of the vertical-axis variable-pitch current turbine, with analyzing on the existing design mentality of blade control mechanism, an optimized law of variable-pitch verticalaxis tidal current turbine was given in terms of the instantaneous moment coefficient of the blade. A method for solving the lift - drag coefficient of Blade by wind - hole test data is given. The optimized kinematic model has a significant reference value for the further development of vertical axis turbine model test.
\end{abstract}

\section{Introduction}

Tide energy is a kind of clean, stable, and rich renewable energy. As the characteristic of simple, non - directional selective, easy-to-install and easy-to-maintain power generation device, the energy capture efficiency of the vertical-axis turbine is always the focus of nowadays research [1]. Experiments and studies have shown that the study of variable pitch system is of great significance to improve the energy capture efficiency of vertical-axis turbine $[2,3]$. And the variable-pitch law is the key part of the variable-pitch system.

In this paper, analysis of hydrodynamic is made and subsequently an optimization model of vertical-axis turbine performance is established, which use the blade instantaneous moment coefficient as an optimization target and blade angle of attack as optimization variable . The range of variation of the design variable is determined by analyzing the lift-drag coefficient and dynamic stall characteristic. Solving the problem by Matlab, the best pitch law is given in terms of the instantaneous moment coefficient of the blade, which provide a new idea on the study of efficiency capture of vertical-axis turbine.

\section{Preliminaries}

\subsection{Hydrodynamic analysis of turbine}

The vertical axis turbine with lift-straight blade is selected as the object of study, and the hydrodynamic study is carried out under the fixed velocity environment [4]

Figure 1 shows the analysis of the force of the impeller. During the constant rotation of the turbine, due to the relative movement between the water flow and the blade, the water flow will produce a resistance $D_{r}$ to the blade opposite to the relative velocity VR and the resistance $\mathrm{D}_{\mathrm{r}}$ is perpendicular to the lift $\mathrm{L}$.

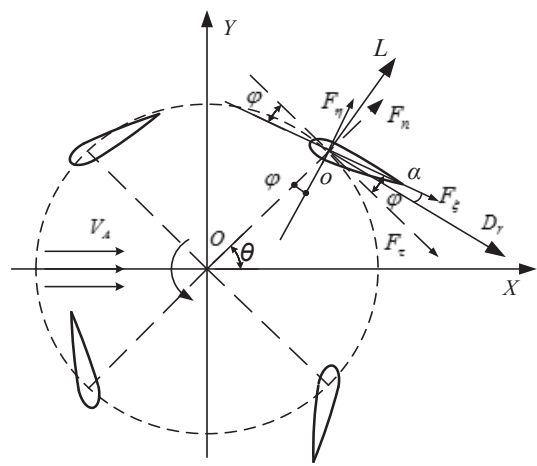

Figure 1. The diagram for the blades and impeller force analysis of the variable-pitch vertical-axis turbine.

Since the straight blades have the same section in the direction of the chord, then a single blade by the lift $\mathrm{L}$ and resistance $\mathrm{D}_{\mathrm{r}}$ can be expressed as the blade unit of the direction of the integral points:

$$
\begin{aligned}
& L=\int_{0}^{b} C_{L} \cdot \frac{1}{2} \rho V_{R}^{2} c d l=C_{L} \cdot \frac{1}{2} \rho V_{R}^{2} c b \\
& D_{r}=\int_{0}^{b} C_{D} \cdot \frac{1}{2} \rho V_{R}^{2} c d l=C_{D} \cdot \frac{1}{2} \rho V_{R}^{2} c b
\end{aligned}
$$

Instantaneous energy utilization factor for a single blade:

$$
C_{t p}=\frac{q(\theta) \cdot \omega}{0.5 \rho V_{A}^{3} D b}=\lambda C_{t q}
$$

In the process of periodic rotation of the blades, the force of both the impeller and the blade are all functions 
of the azimuth angle $\theta$. The average torque coefficient $\mathrm{C}_{\mathrm{q}}$ and the average energy utilization factor $\mathrm{C}_{\mathrm{p}}$ expression of the turbine impeller can be expressed as follow:

$$
\begin{gathered}
C_{q}=\frac{\sigma}{2} \int_{-\frac{\pi}{2}}^{\frac{3 \pi}{2}} \bar{V}_{R}^{2} \cdot\left[\mathrm{C}_{L} \sin (\alpha+\varphi)-\mathrm{C}_{D} \cos (\alpha+\varphi)\right] d \theta \\
C_{p}=\lambda \frac{\sigma}{2} \int_{-\frac{\pi}{2}}^{\frac{3 \pi}{2}} \bar{V}_{R} \cdot\left[\mathrm{C}_{L} \cdot\left(1+\lambda_{1}\right) \cos \theta-\mathrm{C}_{D}\left(\lambda+\left(1+\lambda_{1}\right) \sin \theta\right)\right] d \theta
\end{gathered}
$$

Where: $\sigma$ is the blade density,

$$
\sigma=\frac{z C}{\pi D}
$$

It can be seen from the above equation that the parameters such as the tip ratio $\lambda$, the induction ratio $\lambda_{1}$, the relative velocity coefficient $\mathrm{VR}$, the blade lift coefficient $C_{L}$ and the drag coefficient $C_{D}$ and the blade angle of attack $\alpha$ are directly affected the overall average energy utilization coefficient $\mathrm{C}_{\mathrm{p}}$ of the impeller. For the solution of the induced ratio, there are many solving models in the previous research, and the determination of the airfoil drag coefficient is often dependent on the fluid simulation results, which is different from the actual airfoil parameters. A method for solving the lift-drag coefficient by wind-hole test data of lift-drag coefficient is given in this paper.

\subsection{Analysis on lift - drag coefficient of Blade}

Taking the NACA0018 airfoil as an example, the corresponding wind tunnel experimental data points for the lift-drag coefficient and the range of $0 \sim \pi$ the angle of attack under different Reynolds number was shown in Figure 2:

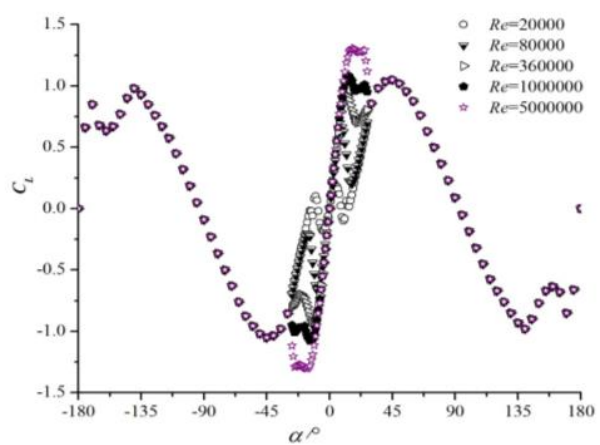

(a) The lift coefficient data points for blade angle of attack

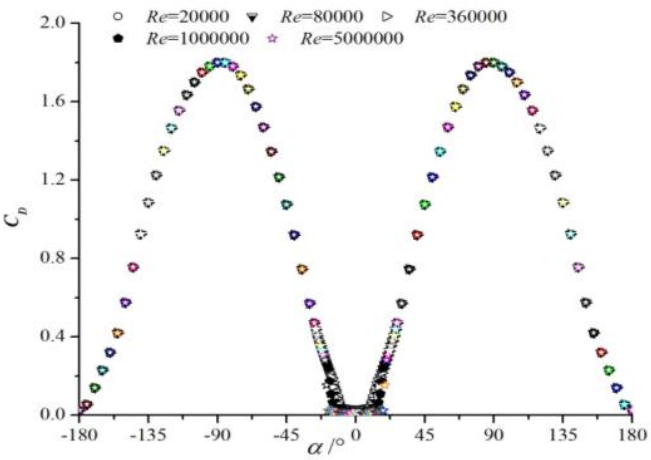

(b) The drag coefficient data points for blade angle of attack

Figure 2. Lift-Drag coefficient blade angle of attack under different Reynolds number
From the above data points, we can see that the Reynolds number and the angle of attack on the airfoil surface are the two main influencing factors of the airfoil. The Reynolds number here is determined by the relative velocity VR between the blade and the fluid, and the relative velocity here is often referred to as the water flow VA. The three-dimensional matrix was established by using the test data and the limited Reynolds number series and the data points under the blade angle of attack. The experimental data matrix is interpolated by cubic spline function in Matlab to solve the blade lift - drag coefficient under the Reynolds number and blade angle of attack.

\section{Optimization model of law of variable- pitch}

Based on the hydrodynamic analysis of the vertical axis turbine, the performance parameters of the vertical axis turbine and the expression of the parameters of the vertical axis turbine are obtained:

$$
C_{q}=\frac{\sigma}{2} \int_{-\frac{\pi}{2}}^{\frac{3 \pi}{2}} \bar{V}_{R}^{2} \cdot\left[\mathrm{C}_{L} \sin (\alpha+\varphi)-\mathrm{C}_{D} \cos (\alpha+\varphi)\right] d \theta
$$

The key factor that determines the output performance of the vertical axis turbine is the pitch law. By changing the law of variable pitch, in order to obtain the optimal value under the fixed tip ratio or the average optimum value in the constant speed ratio to solve the performance optimization problem of the turbine. In order to figure out the optimal pitch law of the verticalaxis turbine, the optimal value of the performance at the fixed blade tip ratio is chosen as the optimization target of the performance optimization.

Thus, the above optimization problem is described in function form as follows:

$$
\left\{\begin{array}{l}
S(\varphi(\theta))=C_{P}(\lambda)=\lambda C_{Q}(\lambda)=\lambda \int_{-\pi / 2}^{3 \pi / 2} C_{t q}(\lambda, \theta) d \theta \\
\max S(\varphi(\theta))
\end{array}\right.
$$

The integral process of the average energy utilization coefficient value of the turbine is the integral process of the discrete energy utilization coefficient. The blade position angle may be discretized in one rotation period of the impeller:

$$
\left\{\begin{array}{l}
\varphi_{i}=\varphi\left(\theta_{i}\right) \\
\theta_{i}=-85^{\circ}+\frac{360^{\circ}}{n} * i \quad(i=0,1,2 \ldots n)
\end{array}\right.
$$

Then the above optimization problem can be expressed as:

$$
\begin{aligned}
& S(\varphi(\theta))=C_{P}(\lambda)=\lambda C_{Q}(\lambda)=\int_{\theta_{0}}^{\theta_{n}} C_{t q}\left(\theta_{i}\right) d \theta \\
& \max S(\varphi(\theta))
\end{aligned}
$$

It is known from the above equation that if the optimal target is maximized, a series of declination of the integral value in one cycle should be the maximum, thus the optimization problem is turn to kind of multi-variable 
nonlinear programming problem. At a specific ratio speed, the optimization target is only the integral of a series of discrete values $C_{t q}\left(\theta_{i}\right)$ in a position of the angular period, so the optimization target the integral value transferred to the discrete value. If the arbitrary position of the instantaneous torque coefficient $C_{t q}\left(\theta_{i}\right)$ comes to maximum, undoubtedly torque coefficient value and the average energy utilization coefficient value of the turbine are fundamentally increased. The designed optimization variable is the variable pitch at any position angle, thus the objective function is also a plurality of discrete values, expressed as follow:

$$
\left\{\begin{array}{l}
\max S\left(\varphi_{i}\right)=C_{t q}\left(\theta_{i}\right)=\bar{V}_{R}^{2} \bar{C} \cdot\left[\mathrm{C}_{L} \sin \left(\alpha_{i}+\varphi_{i}\right)-\mathrm{C}_{D} \cos \left(\alpha_{i}+\varphi_{i}\right)\right] \\
\varphi_{i}=\varphi\left(\theta_{i}\right) \\
\theta_{i}=-85^{\circ}+\frac{360^{\circ}}{n} * i \quad(i=0,1,2 \ldots n)
\end{array}\right.
$$

and:

$$
\left\{\begin{aligned}
\max S\left(\mathrm{C}_{L i}, \mathrm{C}_{D i}\right) & =C_{t q}\left(\theta_{i}\right) \\
= & \bar{V}_{R i} \bar{C} \cdot\left[\mathrm{C}_{L}\left(1+\lambda_{1}\right) \cos \left(\theta_{i}\right)-\mathrm{C}_{D}\left(\lambda+\left(1+\lambda_{1}\right)\right) \sin \left(\theta_{i}\right)\right] \\
\overline{V_{R i}} & =\sqrt{\left(1+\lambda_{1}\right)^{2}+\lambda^{2}+2 \lambda\left(1+\lambda_{1}\right) \sin \theta_{i}} \\
\mathrm{C}_{L i} & =\mathrm{C}_{L}\left(\alpha_{i}\right), \mathrm{C}_{D i}=\mathrm{C}_{D}\left(\alpha_{i}\right) \\
\alpha_{i} & =\alpha\left(\theta_{i}\right) \\
\theta_{i} & =-85^{\circ}+\frac{360^{\circ}}{n} * i \quad(i=0,1,2 \ldots n)
\end{aligned}\right.
$$

It can be seen that the factors that directly affect the optimization target are the position angle $\theta_{i}$, the lift-drag coefficient $\mathrm{C}_{\mathrm{Li}}, \mathrm{C}_{\mathrm{Di}}$ and the relative flow velocity $\mathrm{VR}$. According to the above analysis, it can be seen that in these four factors, except for the position angle $\theta_{\mathrm{i}}$, the second influencing factor is the blade angle of attack $\alpha_{\mathrm{i}}$.

The blade angle of attack at any position is used as the optimization design variable, so that the relationship between the optimization target and the design variable can be expressed more directly.

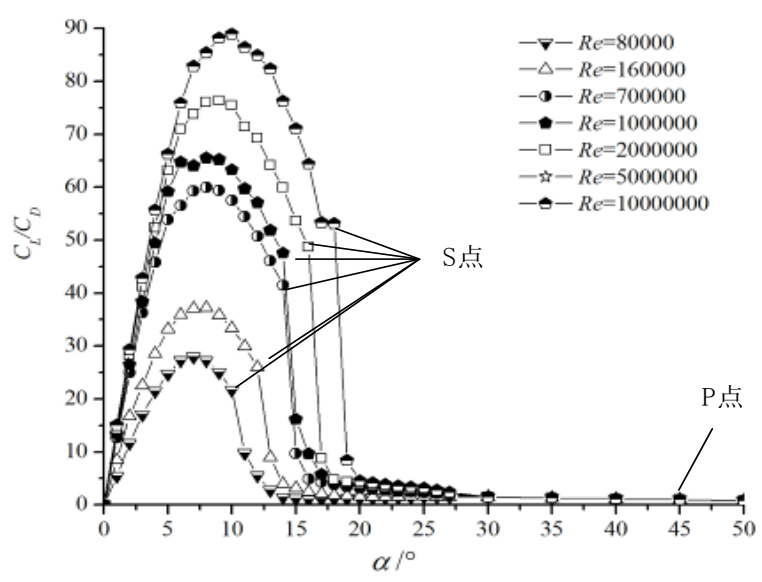

Figure 3. The relationship between airfoil lift - drag coefficient and the angle of attack.

In the design process of the turbine, it is desirable that the airfoil is in good working condition in other words is subject to a large lift and a small drag force. Combined with the airfoil lift - drag coefficient solution given above, the curve of the airfoil lift - drag coefficient with the variation of blade angle of attack is shown in Figure 3. The airfoil lift increases with the increase of the angle $\alpha$, however the airfoil does not produce a large lift in any case. If the blade angle of attack $\alpha$ is large enough to a certain extent, the airfoil coefficient ratio will appear a sharp decline in the phenomenon, called dynamic stall, which was marked point $\mathrm{S}$ in Figure 3 . It can be seen that, the slope of the airfoil increases with the increase of the Reynolds number, and the slope of the lift - drag ratio increases, and the stall point of the airfoil gradually increases after the process of rising and then slowly descending before the point $\mathrm{S}$, while they are all below 16 degrees.

\section{Simulation result}

The objective function of the above-mentioned vertical axis turbine performance optimization problem is a complex nonlinear function with high nonlinearity, multipeak and univariate. The objective function can not be derived. The design variables are discrete and no fixed population. Traditional research algorithms (such as Newton method, quasi-Newton method, conjugate gradient method, rotation direction method) can not be used. Taking into account the design of this variable range is relatively small, the use of simple enumeration method is adopted in this paper. The enumeration method enumerates all the solutions of the objective function in the feasible solution set, and obtains the optimal solution. This method is very suitable for the variable which is relatively small. And the algorithm is simple, easy to solve.

By Using matlab, when at the specified tip ratio, the blade angle of attack - position data points are figured out at the designed speed.

In the calculation of the performance of the vertical axis turbine optimization model, taking into account of working conditions of the turbine blades, run the set angle range $\left(-16^{\circ}, 16^{\circ}\right)$ of the matlab program,

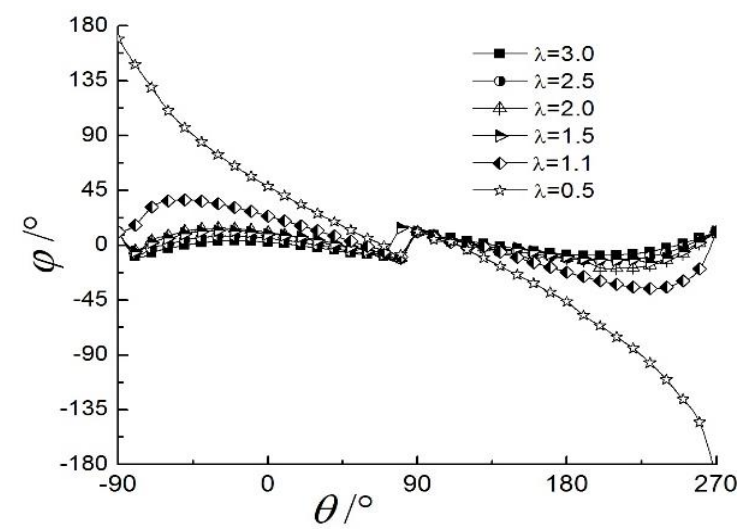

Figure 4. Result of the blade angle of attack and position angle by optimized performance model.

It can be seen from Fig. 4 that the angle of attack of the blade with optimized by the performance optimization model is limited to the set range of the stall angle, and the 
angle of attack angle of $90^{\circ}$ and $-90^{\circ}$ changed dramatically, the variation of the two intervals is about the point $\left(90^{\circ}, 0\right)$ symmetry.

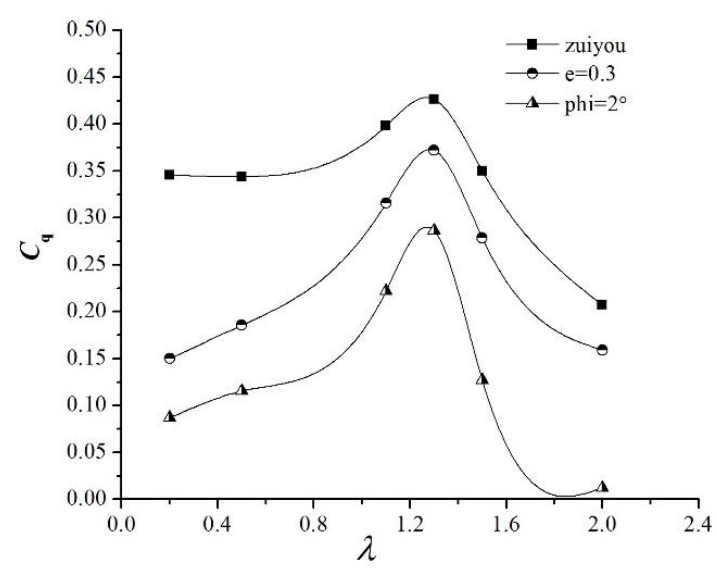

Figure 5. Comparison of $\mathrm{Cq}-\lambda$ curves under different pitch law.

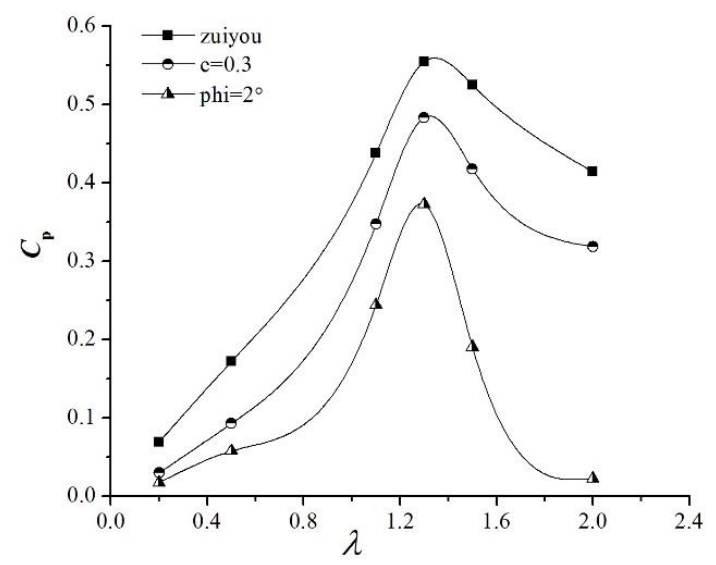

Figure 6. Comparison of $C_{p}-\lambda$ curves under different pitch law.

By comparison, when the tip ratio is within the range of $0 \sim 2$, it can be seen that the solved pitch law is higher than the starting torque under the other two pitch laws, the maximum angle of the average energy capture rate is up to 0.554 , which is better than that of the reference whose theoretical analysis is 0.47 . In the result, the correctness of the performance optimization model of the vertical-axis turbine is verified.

\section{Conclusion}

Based on the analysis of the hydrodynamic performance of the vertical axis tidal power turbine, the instantaneous moment coefficient of the blade is used as the optimization target while the blade angle of attack is the design variable. The model is used to obtain the blade speed. The instantaneous angle of attack of the blade with the best moment coefficient and the variation curve of the energy utilization coefficient under the fixed angle relatively to the conventional cycloidal turbine and the fixed angle turbine.

The energy capture efficiency of the vertical-axis turbine largely depends on the variation pitch law. In this paper, the turbine performance optimization model is established and the optimized pitch law is finally given, which improve the average energy capture efficiency of the turbine to the max 0.554 . It undoubtedly provides the necessary theoretical basis for further research on the hydraulic performance of turbine.

\section{Acknowledgment}

The research is supported by Harbin Applied Technology Research and Development Project:

(NO.2015RQXXJ016);

Harbin Engineering University of basic university basic scientific research business special funds: (HEUCFP201606)

\section{References}

1. Z. Liang, Journal of Hydroelectric Engineering, 35,1-15(2016)

2. P. Koschorrek, C. Siebert, A. Haghani, T. Jeinsch, IFAC-Papers ,48,187-183(2015)

3. J. Yuan, HEU Press, 1,3-4,(2008)

4. H.Wang, I.S., Y.H. Lee, and S.J. Kim. Applied Energy,86(9),1532-1540,(2009)

5. U.Schubert, P. Lampe, IFAC-Papers OnLine ,49, $557-561(2016)$ 